\title{
The Application of Image Processing in Liver Cancer Detection
}

\author{
Meenu Sharma*, Rafat Parveen \\ Dept. of Computer Science, Jamia Millia Islamia \\ New Delhi, India
}

\begin{abstract}
Hepatic cancer is caused by the uncontrolled growth of liver cells, an HCC is the most common form of malignant liver cancer, accounting for 75 percent of cases. This tumor is difficult to diagnose, and it is often discovered at an advanced stage, posing a life-threatening danger. As a result, early diagnosis of liver cancer increases life expectancy. So, using a digital image processing method, we suggest an automated computer-aided diagnosis of liver tumors from MRI images. Magnetic Resonance Imaging (MRI) images are used to identify liver tumors in this case. The image goes through image preprocessing, image segmentation, and feature extraction, all of which are done within the layers of an Artificial Neural Network, making it an automated operation. To make the edge continuous, this operation combines two processes: edge and manual labeling. On the basis of statistical characteristics, tumors are often divided into four categories: cyst, adenoma, hemangioma, and malignant liver tumor. The aim of this proposed technique is to automatically highlight and categorize tumor regions in Magnetic Resonance Imaging images without the need for a medical practitioner.
\end{abstract}

Keywords-Liver cancer; digital image processing; magnetic resonance imaging; early stage

\section{INTRODUCTION}

Every day, billions of cells in our body multiply to create new cells. The newly created cells take up the area previously occupied by dead cells. Cells primarily combine to form tissues, which then combine to form organs [1]. As a result, in some abnormal cases, cells divide faster than the body requires, resulting in lumps or growths that are commonly referred to as tumors [2]. Tumors are irregular tissue growth caused by uncontrolled cell proliferation. They serve no physiological purpose and may be cancerous (malignant or metastases) or non-cancerous (benign) [3]. A benign tumor does not have the ability to spread to other areas of the body, while a malignant tumor does. Malignant tumor begins in the liver and grows on the surface or inside the liver. Primary cancer is described as cancer that begins in a tissue or organ, and primary liver cancer refers to tumors that occur from the liver itself. Hepatocellular Carcinomas is the most common form of malignant liver cancer [4]. We proposed a simple cancer detection approach based on digital image processing in this research paper.

Digital image processing is the method of processing images using computers and the necessary algorithms. Image processing is an evolving and growing industry of medical applications. The tumor area is detected using a computed tomography (CT) scan and a magnetic resonance imaging (MRI) scan image of liver cancer. Magnetic resonance imaging

*Corresponding Author.

e-mail:- meenusharma2275@gmail.com
(MRI) is a much safer technique than CT scan for avoiding ionizing radiation while still providing a better visualization picture of soft tissue. Detecting and analyzing tumors will assist doctors in providing better care to patients and reducing mortality rates [5]. There are three major stages in detecting liver cancer. Image preprocessing, image segmentation, and tumor area highlighting are all part of the process. Image resizing, image contrast enhancement, noise removal filters, and image imperfections are all examples of image preprocessing. This phase is critical in cancer detection because even a slight deviation caused by imperfections or noise can have a significant detection impact [2]. In a decisionoriented application for image segmentation, pixels of an image are correctly categorized. Image segmentation techniques include threshold-based, edge-based, clusteringbased, neural network-based, and others. Image segmentation aims to make an image's representation more relevant and easier to analyze by simplifying or tuning it [6]. In conventional clinical practice, 3-D organ analysis is done manually, which takes a long time. Automated methods have a number of benefits over manual or interactive approaches. Automated methods are more efficient and do not necessitate the intervention of a person [3]. We used the Artificial Neural Network (ANN) algorithm to automate the tumor detection process in this study.

Artificial neural networks are a form of computation inspired by the human brain and nervous system (ANNs). ANNs have been used to perform cognitive tasks usually done by the brain, such as facial recognition, learning to speak and understand a language, recognizing handwritten characters, and determining if the same object is a target viewed from different angles. However, the number of applications for ANNs is the, and they have recently been used successfully in digital image processing [7].

In this analysis, we expect to segment a tumor into a liver, making it easier for the surgeon to see the tumor and treat it. The MRI images are taken in this analysis, and then the segmentation processes are applied to the liver image in order to locate, extract, and further identify liver disease [8]- [10]. We also show how to use image processing to automatically segment CT liver images and remove tumors, as well as categorize tumors into four groups: liver cancer (hepatoma), adenoma, cyst, and hemangioma, with analysis focused on statistical features without the intervention of humans, though keeping in mind that the manual method of testing data sets is time consuming and less accurate than computer-based analysis, and cost is also a consideration [11] - [20]. 
The following is how the paper is organized: Section 2 contains a detailed description of the proposed methodology. Section 3 discusses the findings and their discussion, and Section 4 concludes.

\section{MATERIALS AND METHOD}

The aim of this study is to process and analyze MRI scan images in order to determine if they contain cancer cells or not, and if they do, which type of cancer cells they are. These images come from the Cancer Genome Atlas (TCGA) database, which is open to the public. To process the files, the experimentation procedure employs the MATLAB R2018 program. The segmentation and extraction process depicted in Fig 1 elucidates the overall methodology.

The dataset for this study includes MRI scan images of liver cancer, as well as five images of adenoma, cyst, and hemangioma. Image preprocessing was performed because the images framed by the MRI scan have some noise and discrepancy. The picture is normalized in this step by minimizing noise, changing contrast and scale, and eliminating blurriness all at once. The image's noise and blurriness will slow down the process.

Since the cancer cell is now considered a region of concern, segmenting the liver alone from an abdominal MRI image is complicated since the image contains other organs such as the kidney, spleen, and pancreas that are very close to the liver and have similar intensities. The experiment uses image segmentation based on edge to collect only the liver component and examine the cancer cell. To make the edge continuous, manual labeling is performed. The segmented image, which depicts the extracted liver with cancer cell, is now used to detect cancer cells. The function is extracted by cropping the region of interest, and the k-means algorithm is used to evaluate and judge if the given image is cancer cell or not. Table 1 shows the clusters formed as a result of the abovementioned procedure. The number of pixels must be calculated for statistical analysis of the affected portion, which is done using MATLAB software.

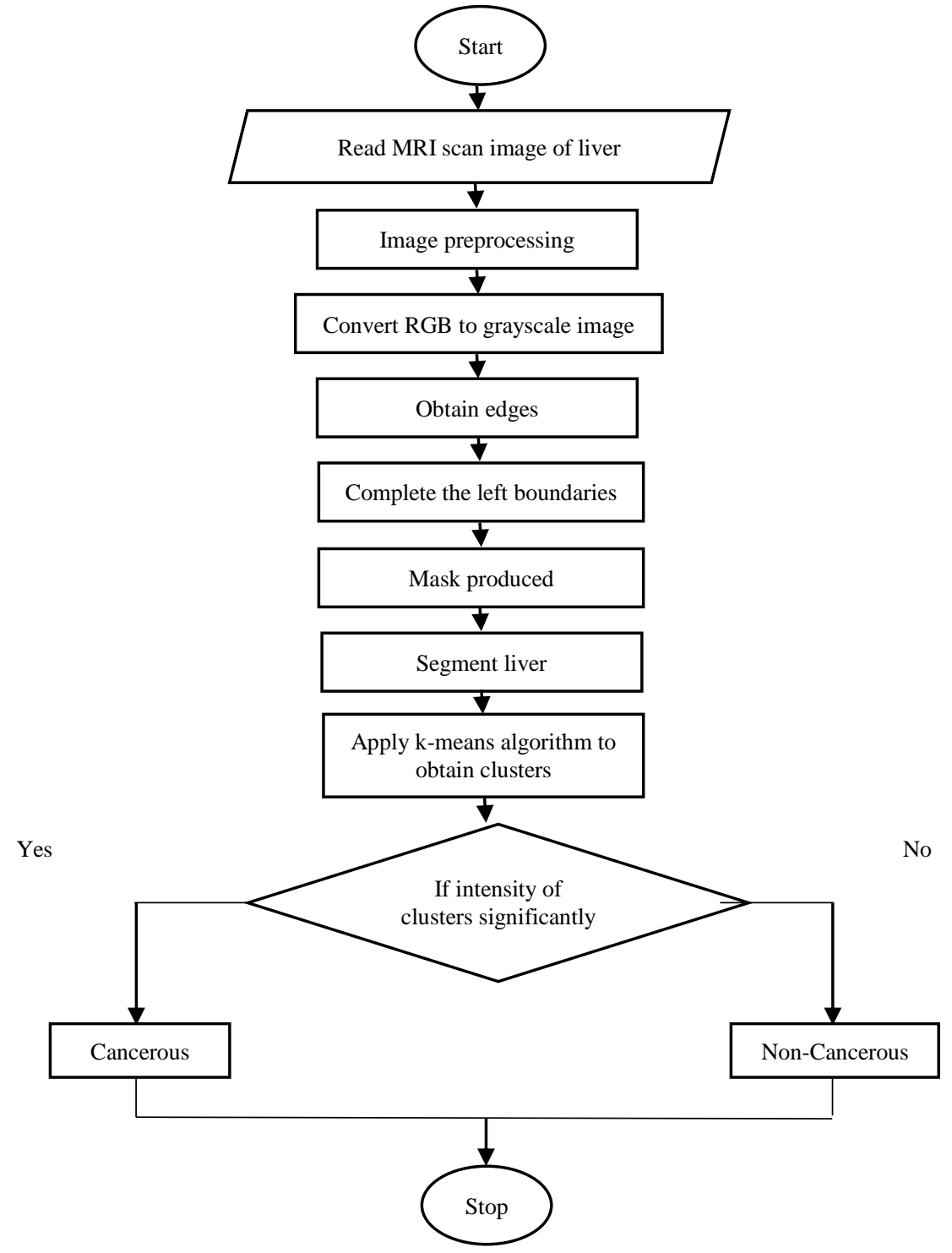

Fig. 1. Proposed Methodology Employed to Segmentation and Tumor Extraction. 
TABLE I. ShOWS THE SEgmentation Result

\begin{tabular}{|c|c|c|c|c|c|}
\hline Sr. No & Original Image & Mask & Liver & k-means segmentation & Extracted feature \\
\hline 1 & & & & $d$ & $d$ \\
\hline 2 & & & & 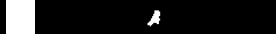 & A \\
\hline 3 & & & & $\forall$ & 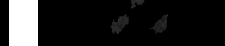 \\
\hline 4 & & & & $\theta$ & $\Rightarrow$ \\
\hline 5 & & & & $d$ & 4 \\
\hline 6 & & & & & 1 \\
\hline 7 & & & & & 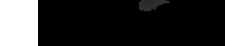 \\
\hline
\end{tabular}




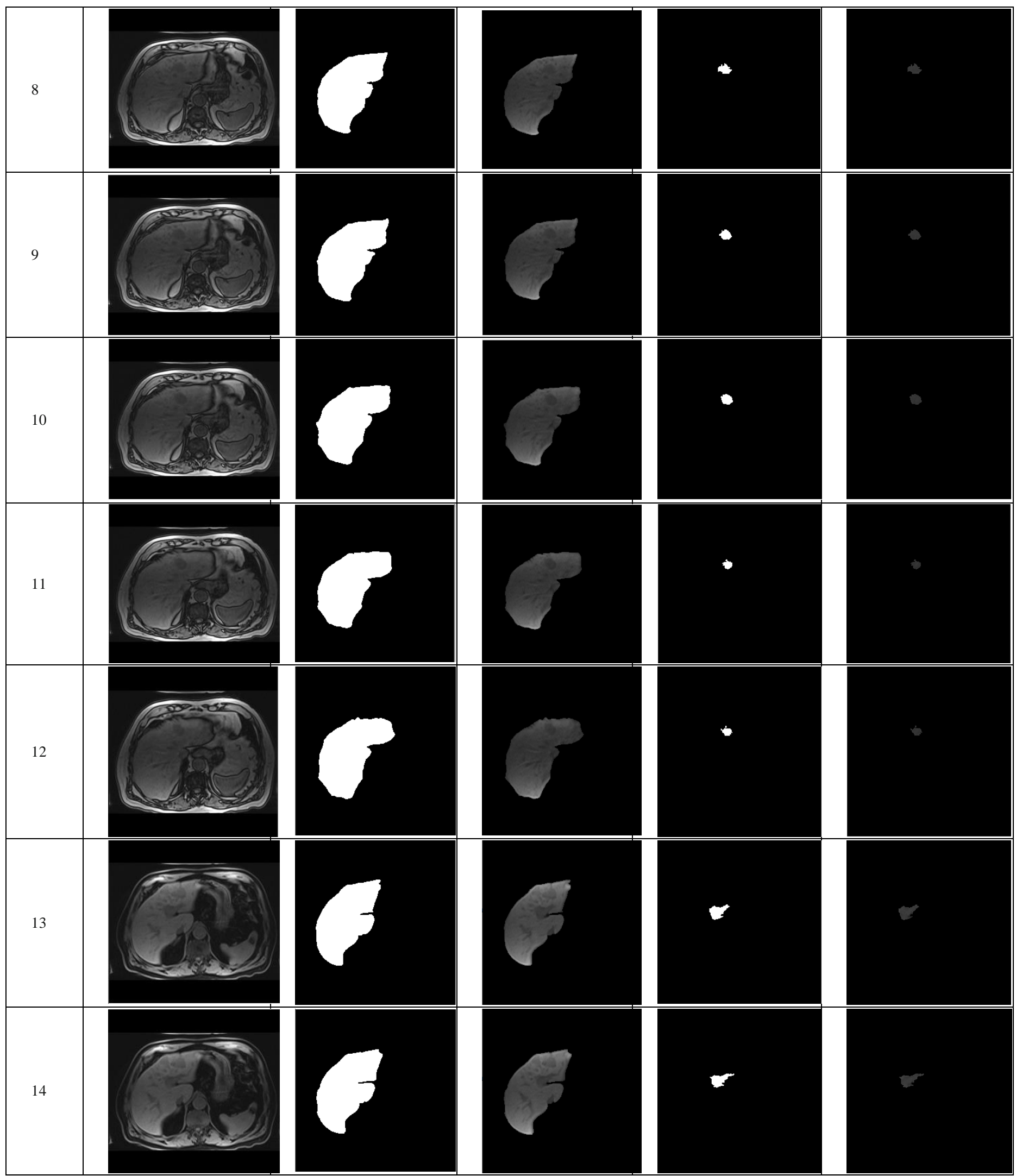




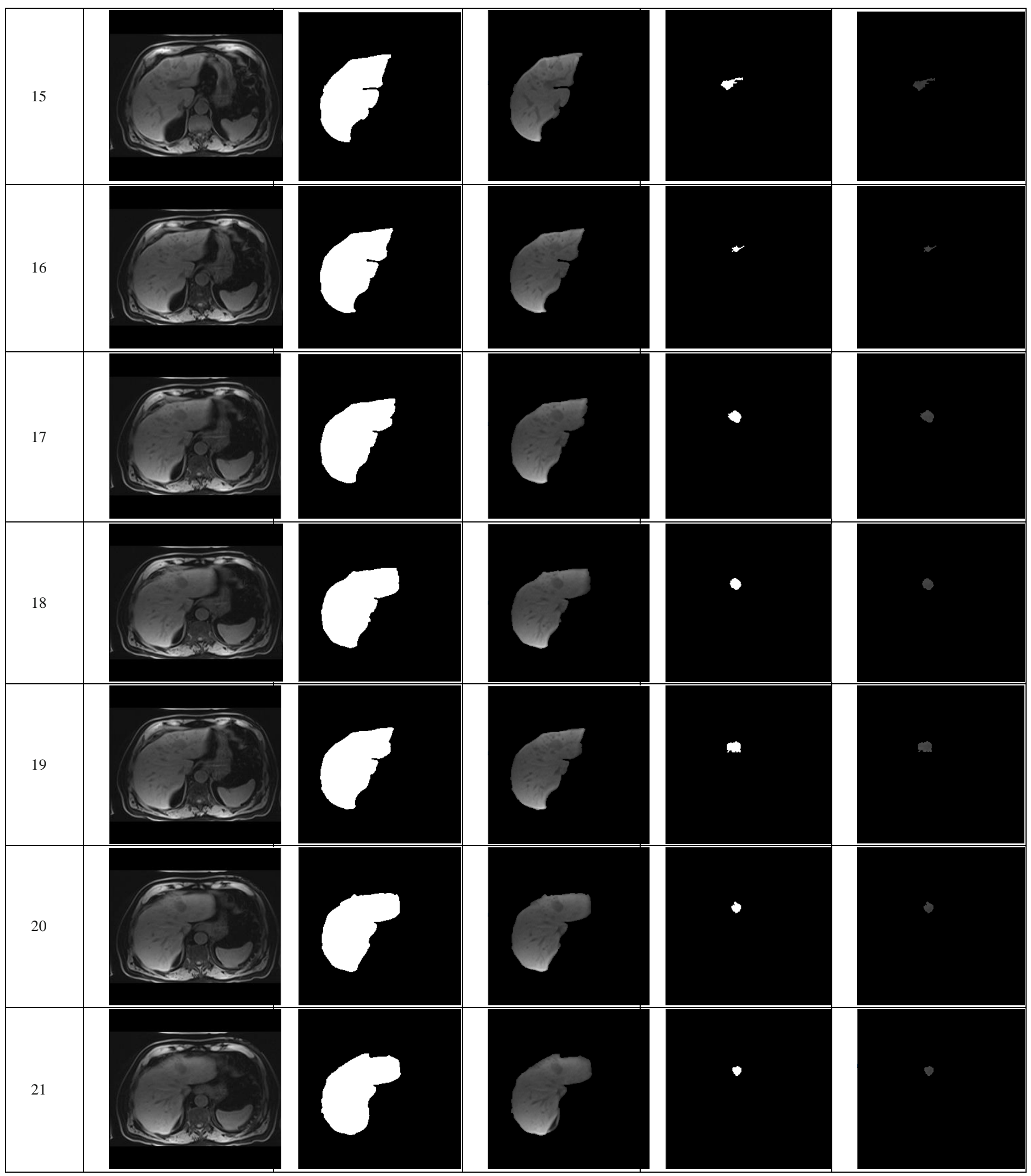




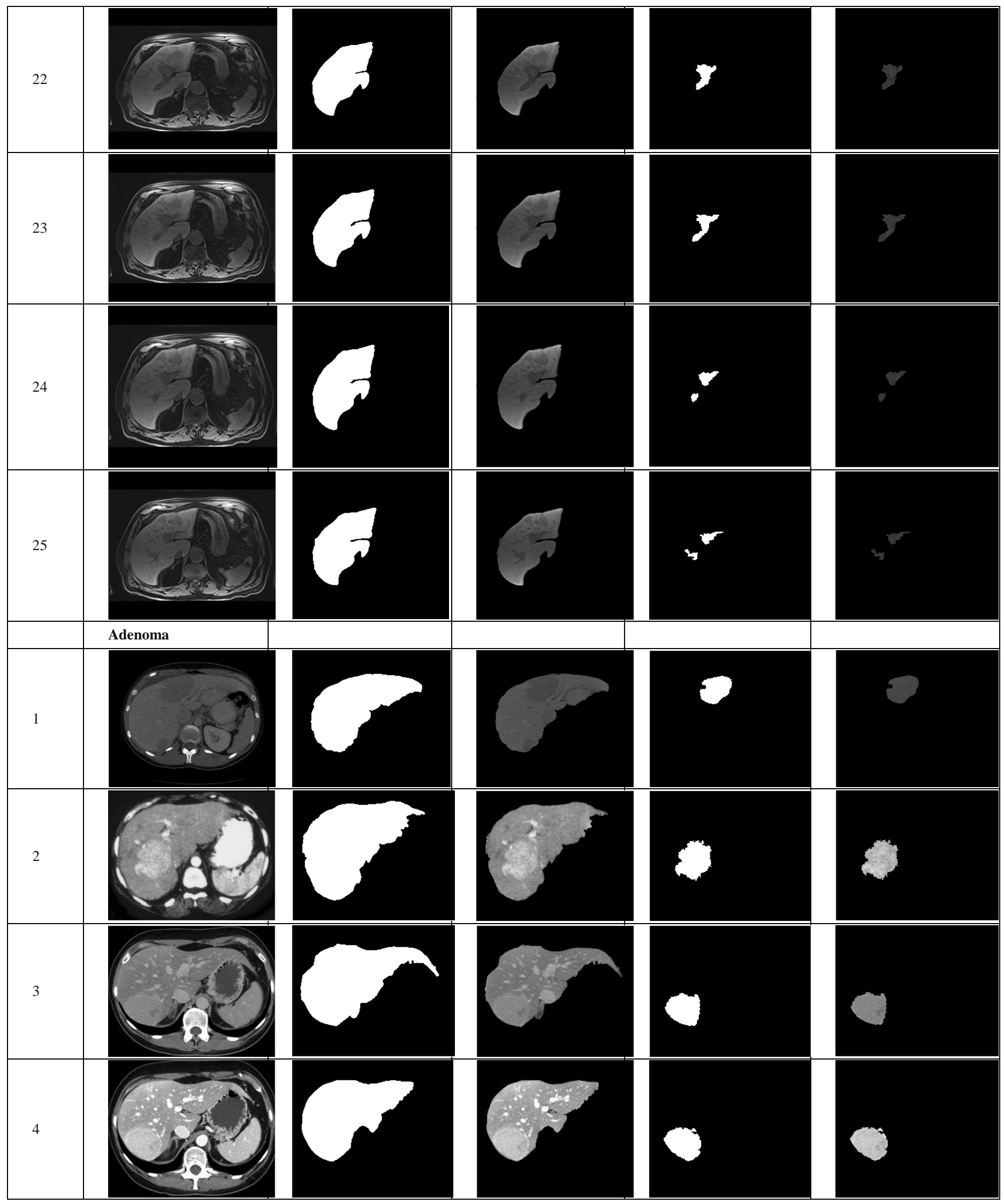




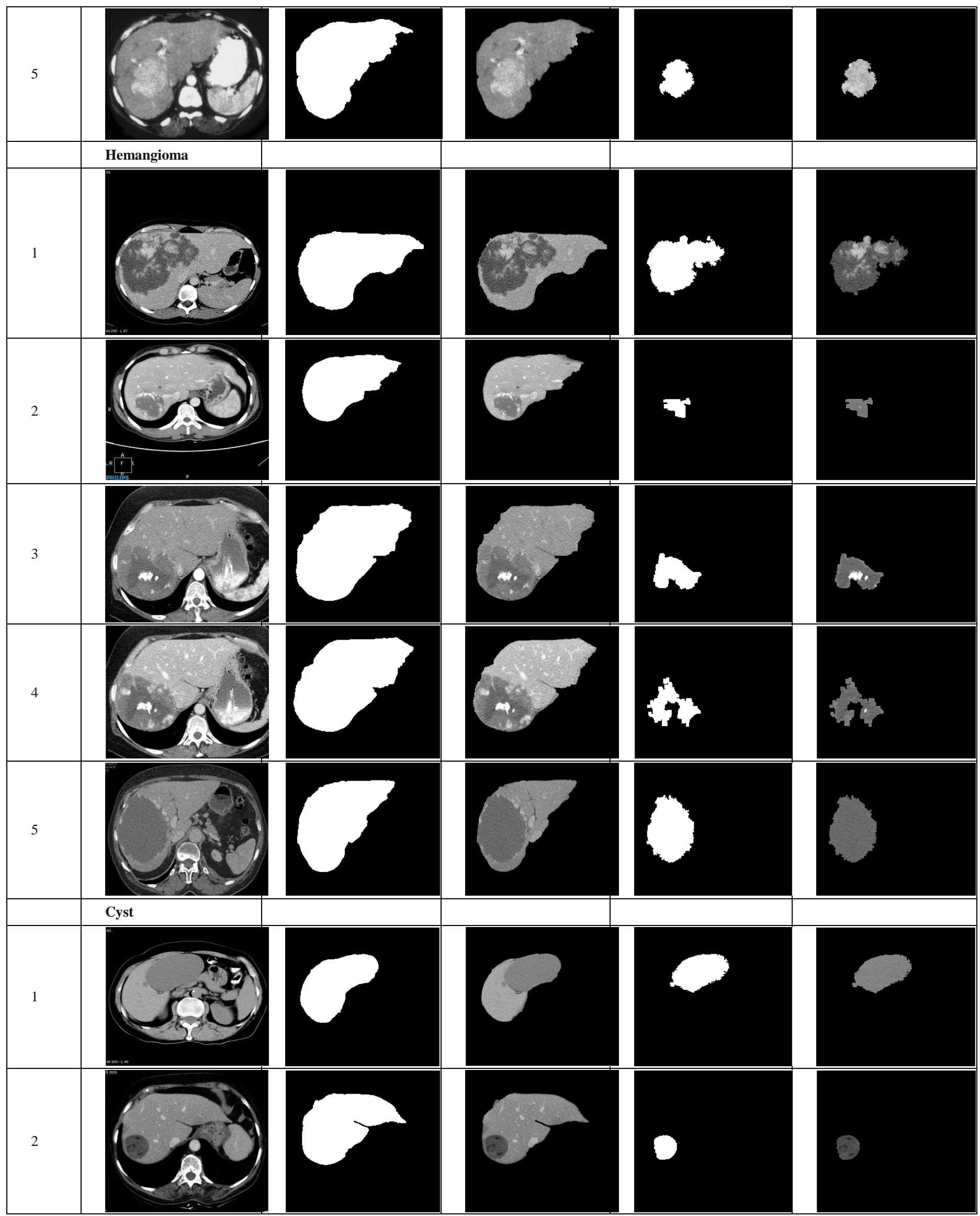




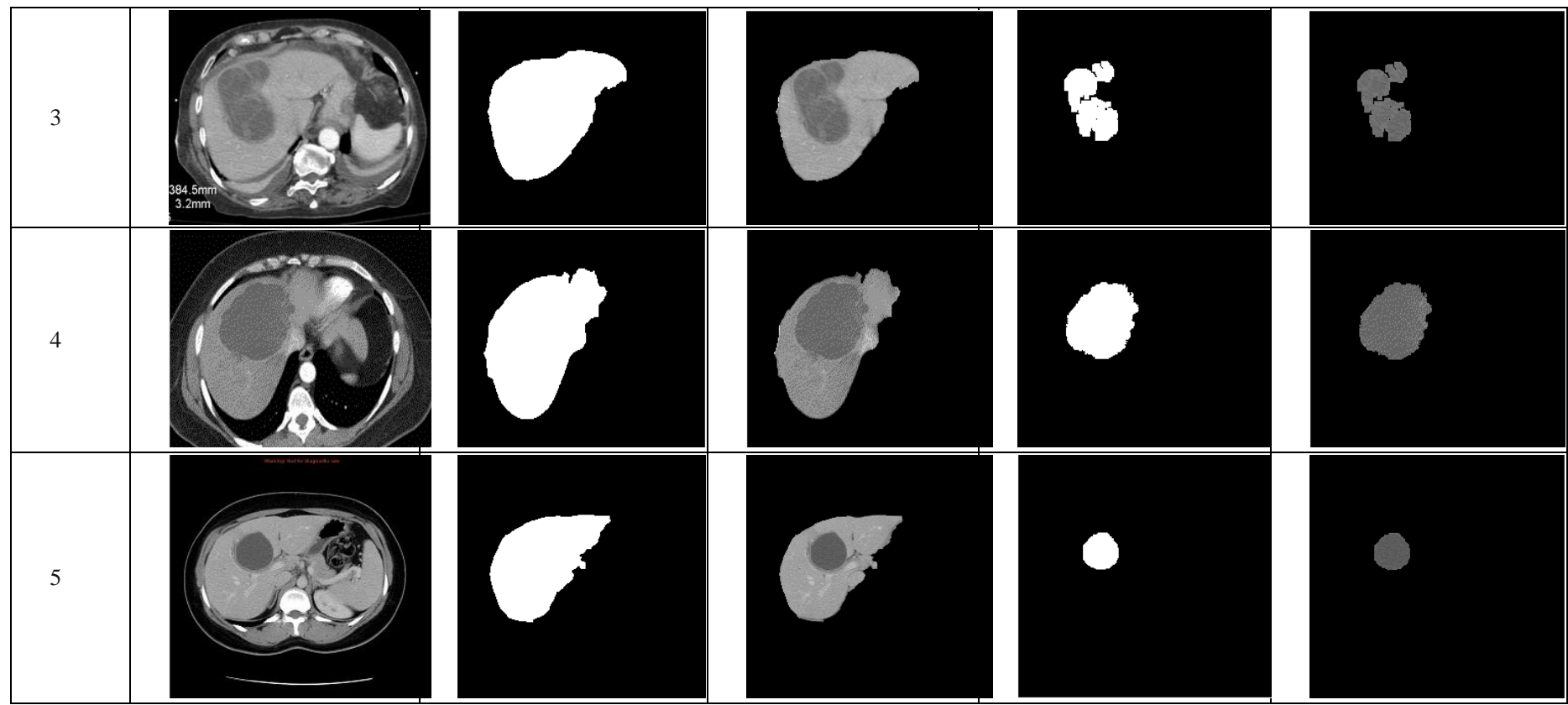

Artificial neural networks are used to automate cancer cell identification and further categorization in liver cancer, cyst, hemangioma, and adenoma. Artificial neural networks (ANNs), also known as neural networks (NNs), are computer structures that are loosely based on the biological neural networks that make up animal brains. The artificial neural network feedforward neural network was used in this study. It is made up of layers of neurons (nodes), such as input, hidden, and output layers. Nodes in neighboring layers have connections or edges connecting them. The input layer is made up of input nodes that provide information from the outside world to the network. This layer includes an MRI scan that is analyzed to determine if it contains cancerous cells and, if so, further categorization. There are no direct relations between the hidden nodes and the outside world.

They perform calculations and send data from the input nodes to the output nodes. While a feedforward network can only have one input layer and one output layer, a hidden layer is formed by a group of hidden nodes. A feedforward network may have zero or several hidden layers. Different masks are saved as hidden nodes in different hidden layers, and the expected edge is compared to all the masks stored in hidden layers, with the highest matching being chosen. The output nodes, collectively known as the "output layer", are in change of computing and distributing network information to the outside world. As seen in Fig 2, this layer automatically generates the segmented liver.

Then, on a segmented liver, use the k-means clustering algorithm to predict the cancer-affected area. As shown in Fig 3 , tumor analysis and categorization were performed on the basis of statistical features into four categories: cyst, liver cancer, adenoma, and hemangioma.

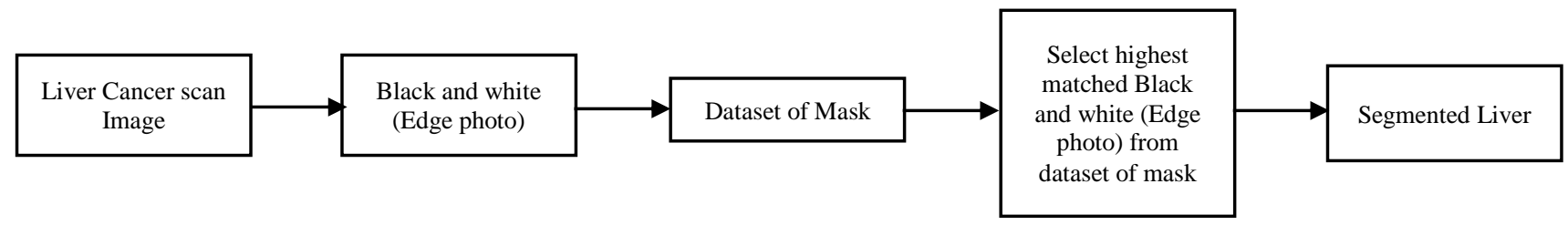

Fig. 2. Automate Segmentation.

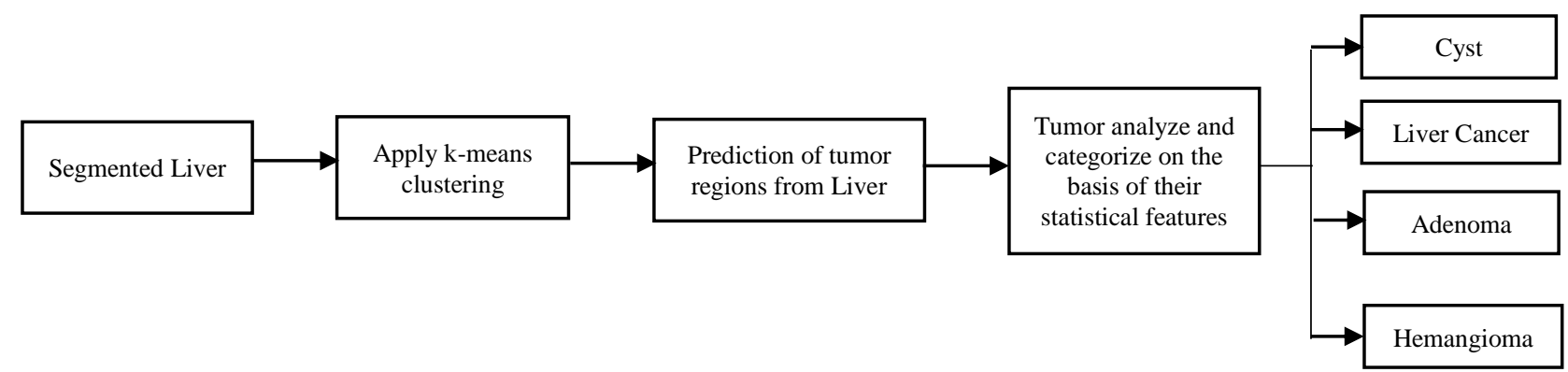

Fig. 3. Categorization of Tumors on the basis of Statistical Features. 


\section{RESULTS AND DISCUSSION}

One of the most difficult challenges confronting today's researchers is cancer detection. Despite extensive study, there is still a lack of an accurate model since detection is a multidisciplinary role that depends on a variety of parameters. The advancement of accurate cancer detection techniques at an earlier stage has been the subject of extensive research. This paper proposed a novel method for the early detection of cancer that is comparatively precise, less time consuming, and simple to calculate.

From the TCGA database, an MRI scan dataset was downloaded. After the liver segmentation preprocessing is completed, a mask for all images is generated. All of these masks are held in a hidden layer at various nodes. This mask was created using an edge, and since it is discontinuous, manual marking was used to make it continuous. To distinguish cancerous from non-cancerous regions, the Kmeans clustering algorithm was used, followed by statistical analysis. Skewness, Kurtosis, Energy, Entropy, Standard deviation, Eccentricity, and Circularity are used in statistical analysis.

When all of the image's textures were analyzed, the average value was determined for all cases of liver cancer, adenoma, hemangioma, and cyst, and then the calculation of the average was obvious by looking at the data in the form of tables. The Hemangioma has the highest values for Skewness, Entropy, and standard deviation, as can be seen in the table. Also, in the Hemangioma case, the energy and circularity are at their lowest levels as compared to other cases. The cancer of the liver has the highest energy value, indicating that it is mild and has less gray color. Since entropy is inversely proportional to energy, liver cancer has the highest energy value. As a result, it has the lowest entropy value.

The skewness gives a visual representation of the textural symmetry. As a result, the Adenoma has the smallest value, and the texture has the least symmetry. This means the texture is irregular and non-homogeneous, much like kurtosis. When it comes to circularity, only a circle has a circularity of one, while every other form has different value than one. The cyst has the highest circularity value, because its form is more circular, while the Hemangioma has a lower circularity value. If the eccentricity value is one, the shape appears to be a line segment, and if it is less than one, the shape becomes more inclined toward a circle. Liver cancer has the highest Eccentricity value, indicating that its shape is more like a line section, while Adenoma has the lowest Eccentricity value, indicating that its shape is more like a circle.

The artificial neural network algorithm is used in the following research to do automatic tumor prediction in an image. Real-time MRI scans, used to determine whether a tumor area exists or not, were obtained from patients and fed into the first layer of an artificial neural network for liver segmentation after preprocessing. In addition, the mask is being prepared for real-time MRI. Then compare the masks saved in the hidden layer with the masks from the real-time MRI. After that, the liver is segmented using the mask with the best match. We get segmented liver in the output layer. To distinguish cancerous from non-cancerous regions, the $\mathrm{K}$ means clustering algorithm was used.

The artificial neural network model is trained using all MRI scan images of liver cancer. A total of ten MRI scan images are used in the testing. The classification using an artificial neural network yielded an average result of 0.9 percent; the annotated result is shown in Fig 4.

The working of the proposed technique is depicted in this research paper on a single MRI scan, with measured attribute values of Eccentricity 0.7924, Circularity 0.7314, Skewness 0.3816, Kurtosis 1.9987, Energy 0.0049, Entropy 0.0415, and Standard deviation 0.0034 after study. When the computed attributes were compared to the average value of all the features (as shown in Table 2), this MRI scan was classified as Liver cancer, which it actually is. Figure 4 depicts the contrast of a real-time MRI scan's discontinuous mask with a mask stored in hidden layer.
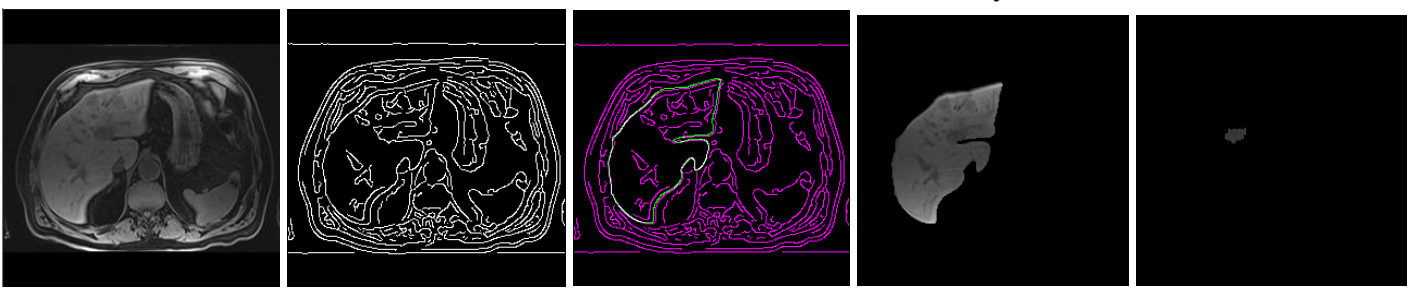

Fig. 4. Shows the Working of our Proposed Method.

TABle II. Average Value of Features for All Cases

\begin{tabular}{|l|l|l|l|l|l|l|l|}
\hline Name & Skewness & Kurtosis & Energy & Entropy & Standard deviation & Eccentricity & Circularity \\
\hline Liver Cancer & 0.5149 & 3.3062 & 0.005583 & 0.0531 & 0.00454 & 0.7223 & 0.6545 \\
\hline Adenoma & 0.4326 & 1.4709 & 0.000497 & 0.4588 & 0.05064 & 0.5867 & 0.5932 \\
\hline Hemangioma & 0.6852 & 2.6214 & 0.000446 & 0.7261 & 0.05382 & 0.6932 & 0.3868 \\
\hline Cyst & 0.4234 & 1.2686 & 0.000589 & 0.4413 & 0.0386 & 0.5945 & 0.6883 \\
\hline
\end{tabular}




\section{CONCLUSION}

Liver segmentation is a difficult task, and automating it is even more difficult, as it involves several steps such as preprocessing, segmentation, and classification. The analogous intensities of other organs such as the spleen, flesh, and muscles are all considered during preprocessing. The artificial neural network algorithm aids in the solution of this problem, automating it through the use of edge and manual marking to create a continuous edge system. The average accuracy rate of the whole liver segmentation using the artificial neural network algorithm is 0.9 percent. Furthermore, an automated method is being developed to identify tumors as benign or malignant, as well as a method to classify perpetual objects using features. It is self-evident that an automated method for classifying tumors as benign or malignant can be useful in object recognition, especially when dealing with medical imaging issues. In addition, a new classification for liver tumors is being considered, which includes more forms of benign liver lesions such as hemangioma, cyst, and adenoma.

\section{ACKNOWLEDGEMENT}

I am thankful to the Indian Council of Medical Research (ICMR), New Delhi, India for providing me funds for doing this research.

\section{REFERENCES}

[1] A. Sharma and P. Kaur, "Optimized Liver Tumor Detection and Segmentation Using Neural Network," no. 5, pp. 7-10, 2013.

[2] R. Aarthi, S. Nivetha, and P. Vikashini, "LIVER CANCER DETECTION USING IMAGE PROCESSING,” pp. 1425-1429, 2020.

[3] A. Raj and M. Jayasree, "Automated Liver Tumor Detection Using Markov Random Field Segmentation," Procedia Technol., vol. 24, pp. 1305-1310, 2016, doi: 10.1016/j.protcy.2016.05.126.

[4] A. Krishan, "Detection and Classification of Liver Cancer using CT Images," no. May, pp. 93-98, 2015.

[5] M. Phil, M. P. Rani, C. Science, and M. Teresa, "Research Article Liver Tumour Detection for Ct Images using Image Processing Techniques Saranya," no. 2015, 2016.
[6] N. Dhanachandra and Y. J. Chanu, "Image Segmentation Method using K-means Clustering Algorithm for Color Image," vol. 2, no. 11, pp. 6872, 2015.

[7] G. Rajesh and A. Muthukumaravel, "Role of Artificial Neural Networks ( ANN )," no. Figure 1, pp. 14509-14516, 2016, doi: 10.15680/IJIRCCE.2016.

[8] A. H. Ali and E. M. Hadi, "Diagnosis of Liver Tumor from CT Images using Digital Image Processing,” vol. 6, no. 1, pp. 685-689, 2015.

[9] J. Ferlay et al., "Cancer incidence and mortality worldwide: Sources , methods and major patterns in GLOBOCAN 2012," vol. 386, 2015, doi: 10.1002/ijc.29210.

[10] K. Ahmed, A.-A.-E. Abdullah-Al-Emran, T. Jesmin, R. F. Mukti, M. Z. Rahman, and F. Ahmed, "Early Detection of Lung Cancer Risk Using Data Mining," Asian Pacific J. Cancer Prev., vol. 14, no. 1, pp. 595-598, 2013, doi: 10.7314/APJCP.2013.14.1.595.

[11] M. Sharma and R. Parveen, "A Complete Summary of Non-Parametric Statistical Methods Used For Biological Microarray Data," no. 4, pp. 4995-5002, 2019, doi: 10.35940/ijrte.D8127.118419.

[12] M. Sharma, "A Comparative Study of Data Mining , Digital Image Processing and Genetical Approach for Early Detection of Liver Cancer," pp. 687-692, 2020.

[13] M. Sharma, "A Novel Digital Image Processing based Mechanism for Liver Tumor Diagnosis," 2021, pp. 58-62.

[14] M. Sharma and R. Parveen, "A Survey on Early Detection of Liver Cancer for Preventive Health Care using Image Processing," vol. 5, pp. 168-173, 2017.

[15] M. Sharma and R. Parveen, "Prior Detection of a Person 's Risk Level of Getting Suffered from Liver Cancer," no. 1, pp. 629-637, 2019, doi: 10.35940/ijitee.A4505.119119.

[16] D. Sharma and G. Jindal, "Identifying Lung Cancer Using Image Processing Techniques," Int. Conf. Comput. Tech. Artif. Intell., pp. 115-120, 2011.

[17] Y. A. Deore, "Efficient Image Processing Based Liver Cancer Detection Method," vol. 3, no. 3, 2014.

[18] A. Verma and G. Khanna, "A Survey on Digital Image Processing Techniques for Tumor Detection," vol. 9, no. April, pp. 1-15, 2016, doi: 10.17485/ijst/2016/v9i14/84976.

[19] W. Wang and C. Wei, "Advances in the early diagnosis of hepatocellular carcinoma," Genes Dis., vol. 7, no. 3, pp. 308-319, 2020, doi: 10.1016/j.gendis.2020.01.014.

[20] L. Ali et al., "Intelligent Image Processing Techniques for Cancer Progression Detection, Recognition and Prediction in the Human Liver." 